\title{
ESTUDO DO COMPORTAMENTO ELETROQUÍMICO DO CONCRETO AUTO ADENSÁVEL E DO CONCRETO CONVENCIONAL SUBMETIDOS A CICLOS DE SECAGEM E MOLHAGEM EXPOSTO AO ATAQUE DE CLORETOS
}

\author{
TABARELLI, ALINE \\ Doutoranda em Ciências e Engenharia de Materiais \\ Universidade Federal de Pelotas \\ Rio Grande do Sul; Brasil \\ tabarellialine@gmail.com
}

\author{
TREIN, KAREN \\ Graduanda em Engenharia Civil \\ Universidade Federal de Pelotas \\ Rio Grande do Sul; Brasil \\ Karen_trein@outlook.com
}

\author{
GARCEZ, ESTELA OLIARI \\ Doutora em Engenharia Civil \\ Deakin University \\ Geelong; Australia \\ estela.o@deakin.edu.au
}

\author{
AVELlanedA, CESAR ANTONIO 0. \\ Doutor em Ciência e Engenharia de Materiais \\ Universidade Federal de Pelotas \\ Rio Grande do Sul; Brasil \\ cesaravellaneda@gmail.com
}

\section{RESUMO}

O Concreto Auto Adensável (CAA) é considerado uma inovação de sucesso na construção civil e proporciona um material mais compacto, com menos poros, sendo considerado uma evolução da tecnologia do concreto. O CAA vem sendo bastante utilizado no Japão e na Europa, mas no Brasil, sua utilização ainda é pequena e são poucos os estudos existentes frente ao comportamento do CAA referente a melhorias principalmente no quesito durabilidade. A corrosão do aço ainda é o principal fator de degradação do concreto armado. A resistividade elétrica do concreto é uma propriedade sensível ao processo eletroquímico que gera a corrosão das armaduras. O presente trabalho tem objetivo avaliar a resistividade elétrica através da técnica de espectroscopia de impedância eletroquímica de dois tipos de concretos de cimento Portland expostos à cloretos: o concreto convencional (CC) e o concreto auto adensável (CAA). O programa experimental consta de corpos de prova de mesma classe de resistência e mesma relação água/cimento, submetidos a ciclos de secagem e molhagem em solução de cloretos por um período de dois anos. Os resultados demostram uma variação na resistividade elétrica onde o CAA possui uma resistividade superior (90\%), e uma degradação inferior (25\%) se comparada com o concreto convencional.

Palavras-chave: Resistividade Elétrica, Concreto, Espectroscopia de Impedância Eletroquímicas, cloretos

\begin{abstract}
Self-compacting concrete (CAA) is an innovation in civil construction and results in a more compact material, with less pores, being considered an evolution of concrete technology. CAA has been widely used in Japan and Europe, but in Brazil, its use is still limited with few studies regarding its behavior especially in terms of durability. Corrosion of steel is still the main reason for reinforced concrete degradation. The electrical resistivity of concrete is a property sensitive to the electrochemical process that causes the corrosion of reinforcement. This work aims to evaluate the electrical resistivity through the electrochemical impedance spectroscopy technique of two types of Portland cement concrete exposed to chloride: conventional concrete (CC) and self-compacting concrete (CAA). The experimental program consists of specimens of the same strength class and the same water/cement ratio, submitted to drying and wetting cycles in a chloride solution for a two-year period. The results indicate significant difference in the electrical resistivity where CAA has a higher resistivity $(90 \%)$, and a lower degradation (25\%) compared to conventional concrete.
\end{abstract}

Keywords: Electrical Resistivity, Concrete, Electrochemical Impedance Spectroscopy, chlorides. 


\section{INTRODUÇÃO}

O vasto uso do concreto como material de construção civil imputa ao meio técnico a preocupação crescente em relação às condições de qualidade do material e aos requisitos de desempenho e segurança estrutural (RIBEIRO et al., 2016). Projetar uma infraestrutura otimizada com melhorias do uso dos recursos, escolha de materiais e processos construtivos atendendo as normas e especificações se torna cada vez mais importante onde desastres anteriores mostram que as falhas na infraestrutura geralmente excede significativamente os danos físicos a esses sistemas (GARDONI E MURPHY, 2018).

Nas últimas décadas, as questões de durabilidade têm ganhado atenção especial e para este estudo o ambiente de projeto com as condições de exposição e as várias influências agressivas ao longo de sua vida útil precisam ser estudadas (ALEXANDER E BEUSHAUSEN, 2019). O processo de degradação do concreto é notoriamente um processo complexo, dependendo de vários fatores, como a composição do material utilizado, o ambiente em que está inserido e ações em que está submetido, como intemperismo e ação humana (SERRALHEIRO et al., 2017). Além disso, durante as fases de produção do concreto, não é possível eliminar todas as fontes de variabilidade nas propriedades dos materiais constituintes da mistura e no próprio processo de preparação do concreto. Nesse sentido, a adoção de controles oportunos sobre as matérias-primas ou sobre os processos produtivos permite conter essa variabilidade dentro de limites pré-estabelecidos (BERTOLINI, 2016).

A corrosão do aço ainda é o principal fator que causa a redução da capacidade de carga e a invalidação de estruturas de concreto armado. Além de diminuir a área efetiva da seção transversal do aço, a corrosão do material provoca uma diminuição da tensão de ligação entre a matriz de concreto e o aço de reforço. Assim, a corrosão devido à diminuição das propriedades mecânicas traz falhas inesperadas no quesito durabilidade e segurança das estruturas de concreto (JIANG et al., 2018; STEFANONI et al., 2018; TIAN et al., 2019; BOURENANE et al., 2019; BALONIS et al., 2019; LI et al., 2019; SHI et al., 2019).

O processo de corrosão induzido por íons cloreto é a causa mais comum de deterioração estrutural e perda de durabilidade do concreto, causando perdas econômicas substanciais em termos de custos de manutenção e reparo (VAN ZIJL GPAG et al., 2018). O período de início de corrosão é afetado pela concentração crítica de cloreto e pelo cobrimento do concreto, ao mesmo tempo em que a porosidade da pasta de cimento influencia diretamente a difusividade do concreto (LUPING E GULIKERS, 2007; ZHANG et al., 2018).

Os estudos de Wang et al. (2017), em estruturas de concreto submetidas a ensaios acelerados em um ambiente agressivo, para análise de durabilidade, demostram que as propriedades mecânicas, como a resistência á compressão, podem diminuir em até $41,6 \%$ onde as conclusões apontam a falha do material, principalmente a danos na estrutura em função do aumento da porosidade e do número das microfissuras.

O concreto auto adensável (CAA) se diferencia do concreto convencional (CC) pela presença de dois novos materiais em sua composição, os quais são materiais finos e aditivos plastificantes ou superplastificantes. Os finos dão coesão para a mistura e resistência à segregação, ocupando espaços que ficariam vagos por agregados maiores, e os aditivos, modificam o concreto quimicamente, reduzindo a quantidade de água para a mistura, e alterando sua viscosidade trabalhabilidade, se moldando automaticamente nas formas sem uso de vibração/espalhamento, com maior durabilidade e resistência (OKAMURA, 1997).

O CAA é considerado uma inovação de sucesso na construção do concreto e no uso rotineiro em mercados de construção competitivos (DE SCHUTTER et al., 2008). É uma tecnologia que fornece muitas soluções para as propriedades do concreto no estado fresco e endurecido e é definida como concreto auto compactante, autonivelante, super trabalhável e não vibratório. As excelentes características de fácil utilização do CAA são de grande atração na indústria da construção tradicional (BUSARI et al., 2018).

Apesar de melhorias na microestrutura dos CAAs, ainda existe carência de informação sobre o material e indicadores de durabilidade (porosidade da água, difusão de cloretos, permeabilidade ao oxigênio) e propriedades adicionais necessárias para uma melhor compreensão da porosidade (ASSIE et al., 2007).

A resistividade elétrica é um parâmetro usado para monitorar a vida útil de estruturas de concreto, que define a habilidade do concreto em transportar cargas elétricas em seu interior, indicador de penetração de agentes agressivos relacionada à permeabilidade do material (MEDEIROS-JUNIOR E GANS, 2017). O nível de resistividade está 
relacionado a microestrutura da matriz de cimento, sua estrutura e a distribuição do tamanho dos poros. É também uma função da concentração de íons e sua mobilidade na solução porosa (ROVNANÍK et al., 2019).

O presente trabalho tem como objetivo avaliar a resistividade elétrica através da técnica de espectroscopia de impedância eletroquímica de dois tipos de concretos de cimento Portland: o concreto convencional (CC) e o concreto auto adensável (CAA) para analisar o mecanismo de degradação frente à exposição a cloretos. O concreto utilizado teve cura de 28 dias e a resitividade foi analisada durante dois anos.

\section{METODOLOGIA}

\subsection{Materiais}

Os materiais adotados na produção dos concretos CC e CAA foram: cimento Portland CP V-ARI, agregado graúdo granítico britado, agregado miúdo areia quartzosa e aditivo superplastificante de alto desempenho. A Tabela 1 apresenta as propriedades dos materiais adotados.

Tabela 1 - Propriedades dos Materiais adotados na produção do CC e CAA

\begin{tabular}{c|c|c|c|c|c}
\hline Propriedades & Cimento Portlhand CP V - ARI & Brita 0 & Brita 1 & Areia 1 & Areia 2 \\
\hline Massa específica $\left(\mathrm{g} / \mathrm{cm}^{3}\right)$ & 3,21 & 2,65 & 2,604 & 2,604 & 2,59 \\
\hline Massa Unitária $\left(\mathrm{g} / \mathrm{cm}^{3}\right)$ & - & 1,35 & 1,51 & 1,548 & 1,28 \\
\hline Módulo de Finura & - & 5,07 & 6,58 & 2,13 & 1,04 \\
\hline Índice de Vazios (\%) & - & 48,68 & 42,0 & - & - \\
\hline $\begin{array}{c}\text { Diâmetro Máximo } \\
(\mathrm{mm})\end{array}$ & - & 9,50 & 19 & 1,18 & 0,30 \\
\hline
\end{tabular}

No CAA, o aditivo superplastificante utilizado foi de terceira geração de policarboxilato (faixa de dosagem: Mínimo 0,2\% / Máximo 2\%). O aditivo é isento de cloretos.

\subsection{Métodos}

O programa experimental consta da análise de corpos de prova de CC e CAA de mesma classe de resistência e mesma relação água/cimento. Em relação aos limites normativos da norma brasileira NBR 6118 (ABNT, 2014) em função da durabilidade das estruturas, este trabalho adotou os parâmetros de projeto mínimos para uma classe de agressividade forte, Classe III (ambiente marinho). Tais considerações dizem respeito a critérios de projeto de modo a garantir o desempenho da estrutura de concreto armado frente a sua vida útil estimada.

O método usado para dosagens do CC foi o método do EPUSP/IPT (HELENE E TERZIAN, 1992) e para o CAA foi o método do Tutikian e Dal Molin (2005). A idade de cura úmida adotada foi de 28 dias para ambos os concretos.

Neste trabalho para o CC o teor de argamassa adotado foi de $51 \%$, o consumo de cimento de $382,6 \mathrm{~kg} / \mathrm{m}^{3}$ e uma relação água/cimento de 0,5. O traço adotado foi de 1:1,918:2,803 (cimento : agregado miúdo : agregado graúdo). Para o CAA o traço adotado foi de 1: 2,520:1,813 (cimento : agregado miúdo : agregado graúdo) com uma relação de argamassa de $66 \%$, com um consumo de cimento de $406,72 \mathrm{~kg} / \mathrm{m}^{3} \mathrm{com}$ a mesma relação água/cimento.

A análise do estado fresco do CC foi baseada na norma NBR 67 (ABNT, 1998) e NBR 12655 (ABNT, 2015) e a análise do CAA na norma NBR 15823-1 (ABNT, 2017). A análise do estado endurecido foi feita através da caracterização da resistência mecânica pelas normas NBR 5739 (ABNT, 2018) e NBR 5738 (ABNT, 2016) para ambos os concretos. A avaliação da porosidade foi feita através do ensaio de absorção de água por capilaridade baseado na norma NBR 9779 (ABNT, 2012). Para tais ensaios, corpos de prova cilíndricos $(10 x 20 \mathrm{~cm})$ foram moldados, desmoldados após 24 h e curados por 28 dias.

As análises da resistividade dos corpos de prova foram calculadas através da técnica de Espectroscopia de Impedância Eletroquímica (EIS) com o auxílio de um potenciostato IVIUM STAT com medidas em potencial de circuito aberto com amplitudes de perturbação no potencial $0,025 \mathrm{~V}$. Os intervalos de frequência adotados foram de $0,05 \mathrm{a} 10^{6} \mathrm{~Hz}, \mathrm{com}$ 5 pontos por década. 
A moldagem dos corpos de prova para a análise do estado endurecido e para análise eletroquímica foram em corpos de prova de iguais dimensões. Na pilha galvânica, para o método eletroquímico, foram adotados dois eletrodos embutidos na amostra, com o eletrodo de aço (CA-50) associado a um eletrodo de referência de grafite (Figura 1). A interface armadura e argamassa do CP possui uma área de exposição de $35 \mathrm{~cm}^{2}$. Os dados foram analisados para um grupo amostral de 9 corpos de prova para cada tipo de concreto.

O processo de envelhecimento acelerado adotado consiste em ciclos semanais de secagem e molhagem a meia altura dos corpos de prova de modo a expor os corpos de prova periodicamente à solução de cloreto, simulando um ambiente de marés (FERREIRA, 2015). Assim, os ciclos semanais promovem a entrada do oxigênio e do eletrólito no concreto, favorecendo o processo de corrosão eletroquímico. A penetração dos íons de cloreto se dá pelas etapas de imersão das amostras em um recipiente contendo uma solução de cloreto de sódio (3,5\%) por 2 dias e após este período secagem por 5 dias em um ambiente controlado (WEI et al., 2018; WU et al., 2017, ANGST et al, 2011). Os ciclos são adotados em ambiente laboratorial para simular as condições ambientais secas e úmidas.
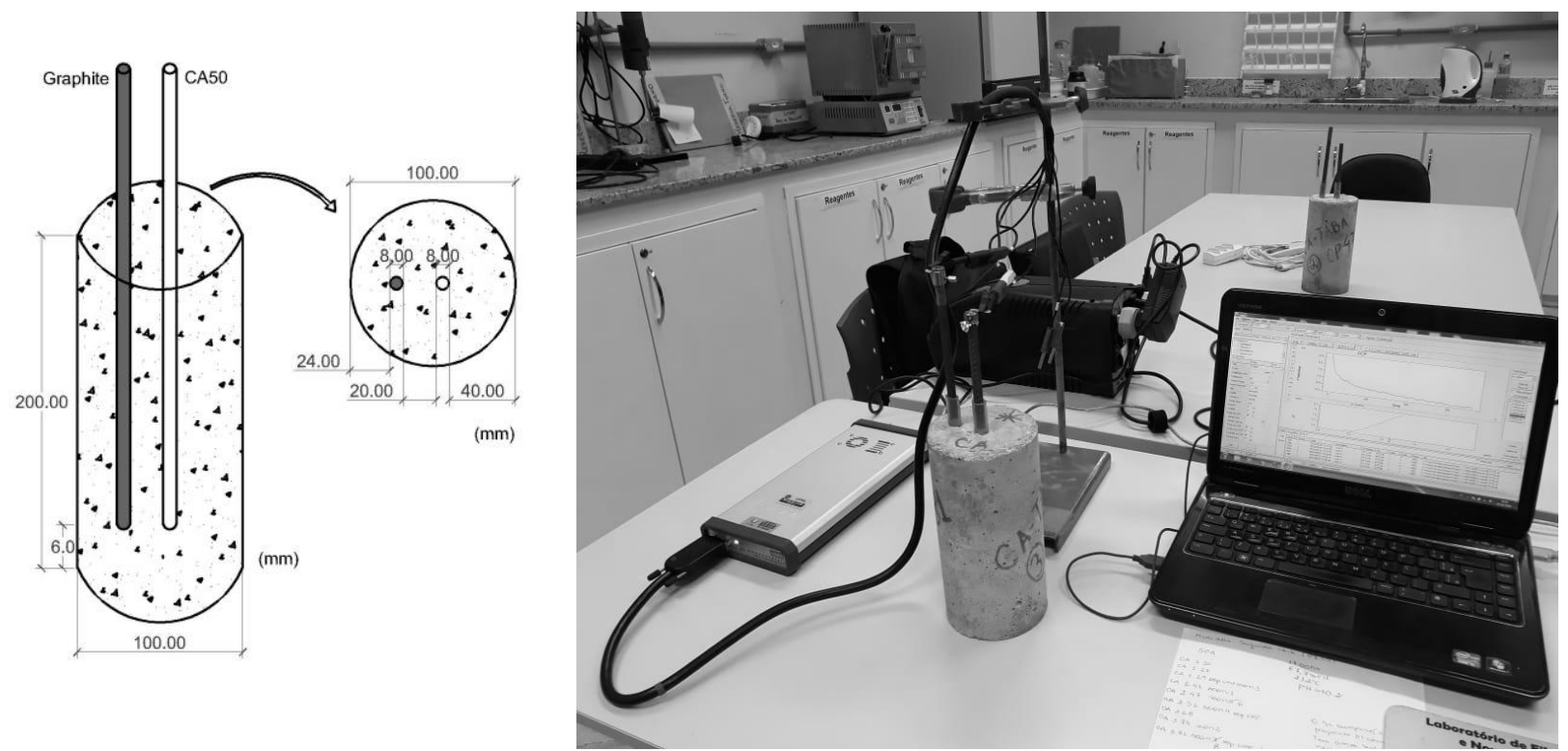

Figura 1: Esquema Experimental para análises eletroquímicas. Corpo de Prova e Medidas (a, b)

O estudo do avanço da penetração de cloretos nos corpos de prova foi baseado na entrada de cloreto por ciclos de secagem e molhagem em uma solução de $\mathrm{NaCl}$ durante dois anos de exposição. As amostras foram rompidas diametralmente após o período de exposição e submetidas a aspersão de uma solução aquosa de nitrato de prata $0,1 \mathrm{~N}$ $\left(\mathrm{AgNO}_{3}\right)$ conforme ensaio colorimétrico (REAL et al., 2015; HELENE et al., 2018). A seção tranversal da amostra de concreto rompida é pulverizada com a solução gerando regiões de coloração distintas que indicam a presença de cloretos (zona esbranquiçada). Após o ensaio, as regiões esbranquiçadas formam medidas a cada $1 \mathrm{~cm}$ conforme descrito na norma ASTM G1:2003. As medidas foram estudadas para um grupo amostral de 9 amostras de cada tipo de concreto.

A análise microscópica foi feita através da técnica de análise de microscopia eletrônica de Varredura (MEV) com um Jeol , JSM-6610LV e através da técnica de caracterização de difração de raio X (DRX) Bruker, D8 Advance localizado no CEME-Sul no campus FURG em Rio Grande.

\section{RESULTADOS E DISCUSSÕES}

\subsection{Estado Fresco e Ensaio Endurecido}

Os resultados das propriedades dos concretos no estado fresco estão apresentados na Tabela 2. Os resultados garantem um concreto convencional com vibração manual e o concreto auto adensável bombeado não vibrado para aplicações em vigas, pilares e pré-moldados de acordo com as especificações. 
Tabela 2 -Análise do Estado Fresco para CC e CCA

\begin{tabular}{c|c|c}
\hline & CC & CAA \\
\hline Slump Test $(\mathrm{mm})$ & 70 & - \\
\hline Cone Abrams $(\mathrm{mm})$ & - & 590 \\
\hline Caixa L $(\mathrm{H} 2 / \mathrm{H} 1)$ & - & 0,77 \\
\hline T500 (segundos) & - & 2,19 \\
\hline Funil V & - & 4,47 \\
\hline Anel J & - & 50 \\
\hline
\end{tabular}

A Tabela 3 apresenta os resultados de resistência mecânica à compressão axial dos CC e CAA, garantido a mesma classe de resistência.

Tabela 3 - Resistência a compressão para CC e CCA

\begin{tabular}{c|c|c|c|c}
\hline & \multicolumn{2}{|c|}{ CC } & 28 dias & 91 dias \\
\hline Idade & 28 dias & 91 dias & 42,35 & 45,99 \\
\hline fcj médio( MPa)* & 42,38 & 46,02 & 1,03 & 1,26 \\
\hline Desvio padrão & 1,26 & 0,89 & 2,42 & 2,74 \\
\hline Coeficiente de variação & 2,97 & 1,95 & \\
\hline
\end{tabular}

fc,j* - resistência média à compressão

Para avaliação da porosidade dos dois concretos, os resultados de absorção de água por capilaridade aos 28 dias segundo a norma NBR 9779 (ABNT, 2012) são apresentados na Figura 2, comprovando que o CAA é um concreto menos poroso se comparado com o concreto convencional.

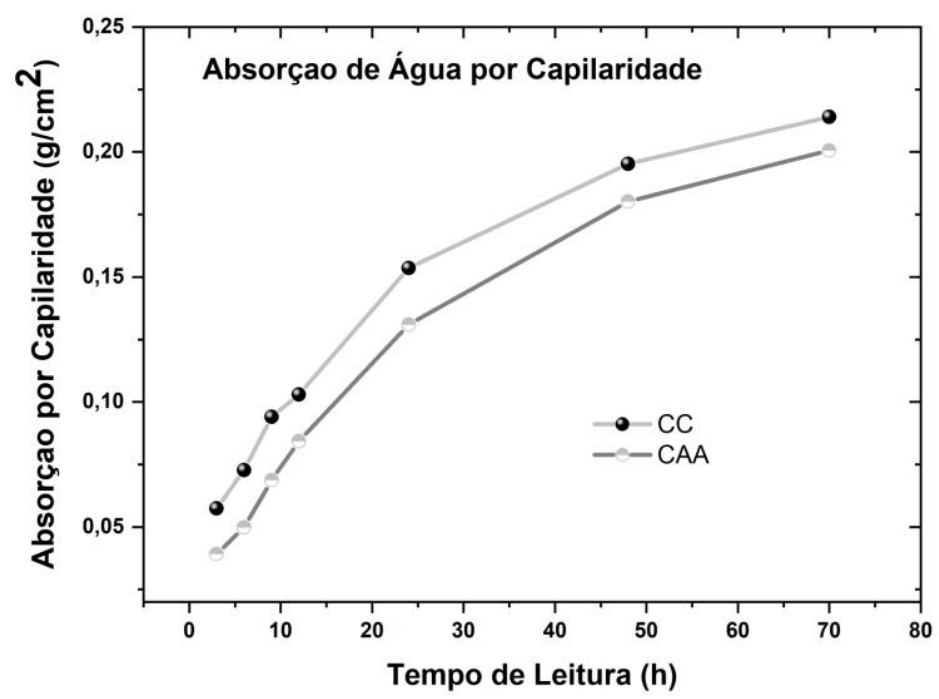

Figura 2: Curva de absorção por capilaridade em função do tipo de concreto

3.2 Análise morfológica e mineralógica

Para verificar a penetração dos íons cloretos nas amostras de CC e CAA foi realizado um MEV com ampliação de 1000 vezes para observar a presença de cristais de $\mathrm{NaCl}$ em uma fase denominada sal de Friedel e análise mineralógica em DRX para sua confirmação. As imagens de MEV e o difratograma de DRX foram observadas após 2 anos de ciclos. As amostras foram preparadas em sólido para o MEV e em pó para o DRX após serem secas em estufa a $100^{\circ} \mathrm{C}$ por 3 dias. Esta fase é resultado da ligação dos cloretos livres ao aluminato tricálcico resultado das fases sólidas do cimento hidratado (GUIRGUIS et al., 2018). A Figura 3 apresenta os resultados do DRX com faixa angular de $10^{\circ}$ a $90^{\circ}$ com passo de 0,05 e tempo de 1 s confirmando a presença da formação do sal de Friedel $(*)\left(\left(\mathrm{Ca}_{2} \mathrm{Al}(\mathrm{OH})_{6} \mathrm{Cl}\left(\mathrm{H}_{2} \mathrm{O}\right)_{2}\right)\right.$ no 
concreto convencional. Nas imagens de MEV (Figura 4 e 5) confirmam a microestrutura dos concretos e a presença do sal no concreto convencional.
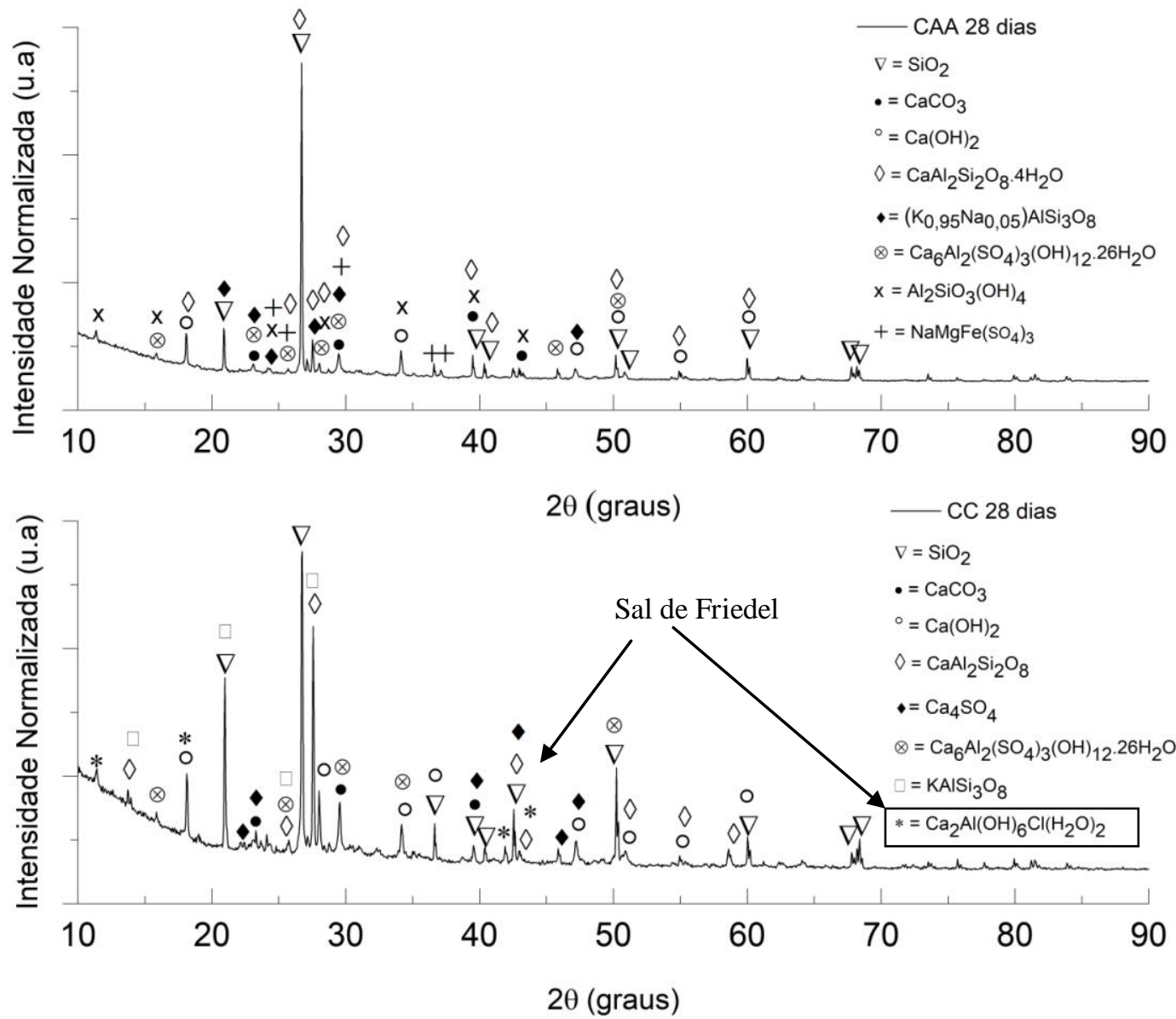

Figura 3: Difratograma de raio $\mathrm{X}$ comparativo dos concretos

A formação do sal de Friedel no CC demostra a presença de íons de cloro nas amostras. Deste modo, os resultados apresentados indicam a penetração dos cloretos no concreto convencional . O sal não foi encontrado no difratograma do CAA para o mesmo período de ciclos de secagem e molhagem de dois anos.

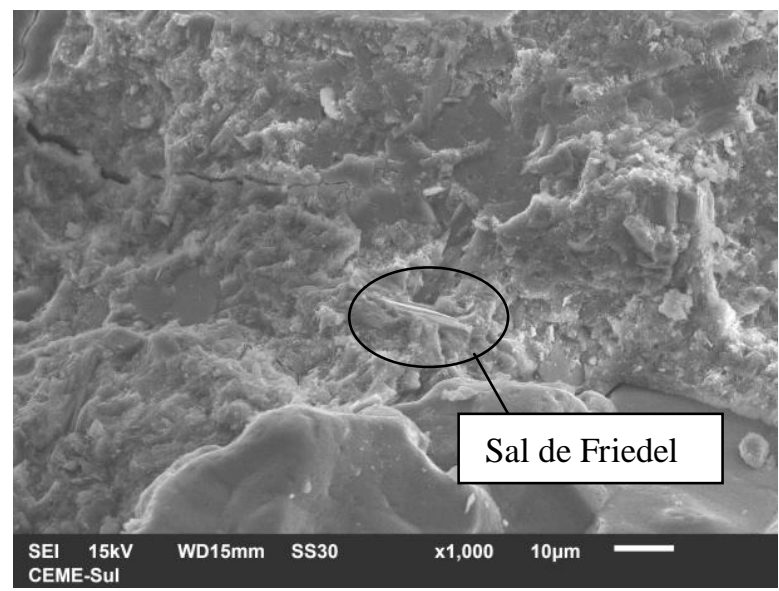

Figura 4: Análise morfológica do concreto convencional exposto às 2 anos de ciclos de secagem e molhagem indicando a presença de sal de Friedel (cristais). Imagem obtida através do MEV 


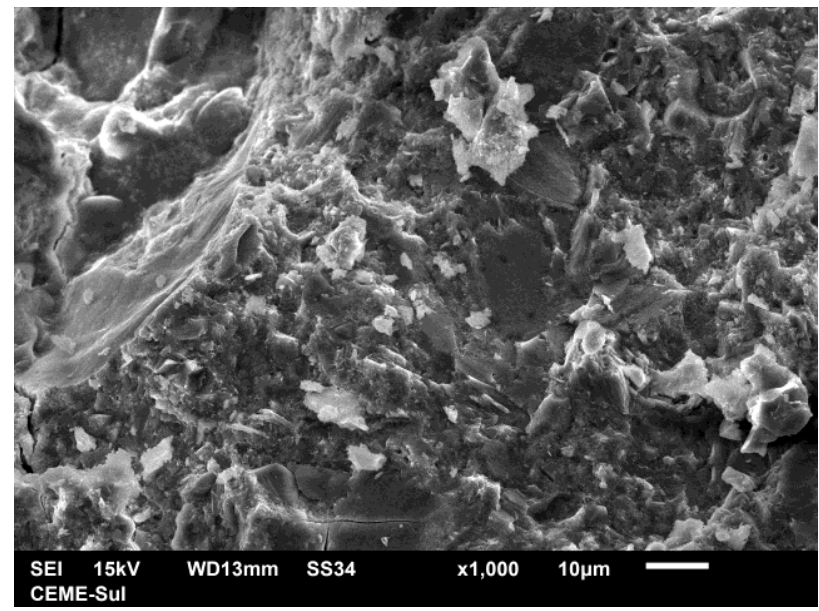

Figura 5: Análise morfológica do concreto auto adensável exposto a 2 anos de ciclos de secagem e molhagem. Imagem obtida através do MEV

\subsection{Penetração de cloretos}

O resultado encontrado no ensaio de penetração de cloretos para ambos os concretos analisados no período de 2 anos foi uma média de $3,55 \mathrm{~cm}$ para o concreto convencional e $2,91 \mathrm{~cm}$ para o concreto auto adensável para os corpos de prova com idade de cura de 28 dias (Figura 6) e submetidos ao processo de envelhecimento acelerado. Este resultado comprova que a penetração dos cloretos foi significativa, principalmente no $\mathrm{CC}$ e praticamente atingindo o limite de norma de $4 \mathrm{~cm}$.

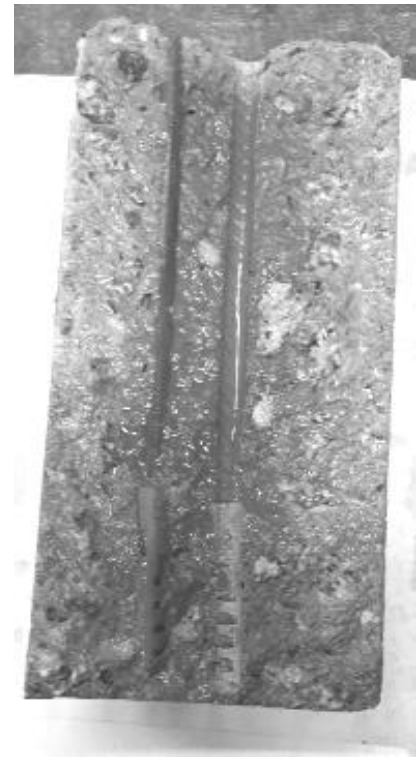

CAA

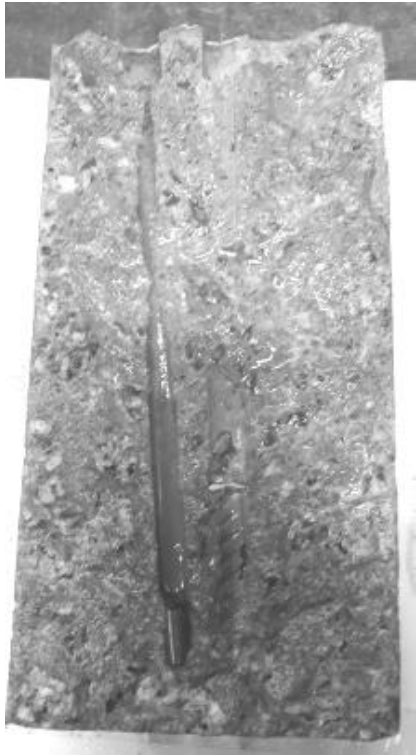

$\mathrm{CC}$

Figura 6: Corpos de Prova de CAA (a) e CC (b) exposto a 2 anos após rompimento e aspersão da solução colorimétrica

\subsection{Análise eletroquímica}

As curvas geradas pelo EIS apresentam comportamentos semelhantes para ambos os concretos, sendo curvas representativas para materiais cimentícios (VENDALAKSHMI et al., 2009; BRAGANÇA et al., 2014; SOHAIL et al., 2020). 
Analisando o comportamento da resistividade elétrica do concreto convencional e do concreto auto adensável, verificase uma resistividade elétrica superior no auto adensável. Os valores da resistividade elétrica geralmente aumentam com as idades durante o processo de hidratação do cimento em condições normais, mas devido à exposição aos cloretos e ao consequentemente processo de penetração de íons cloreto nos concretos, uma redução na resistividade é observada. Essa redução inicia-se aproximandamente no mesmo período, mas com valores distintos (Figura 7).

A Figuras 7 apresenta o diagrama de Nyquist (Impedancia Real x Impedancia Imaginária) em funçao de três períodos distintos. O período inicial é considerado o ciclo antes da exposição, e consequentemente sem penetraçao de cloretos. A resitência elétrica máxima, observada antes da redução da resistividade, aconteceu para os dois concretos após um período de um ano e meio (70 ciclos). Comparando a Figuras 7 verifica-se um aumento de em torno de $60 \%$ de sua resistividade elétrica do CAA em relação ao CC nas primeiras idades, se tornando mais expressivo a medida que o processo de hidratação vai ocorrendo chegando a quase $100 \%$.
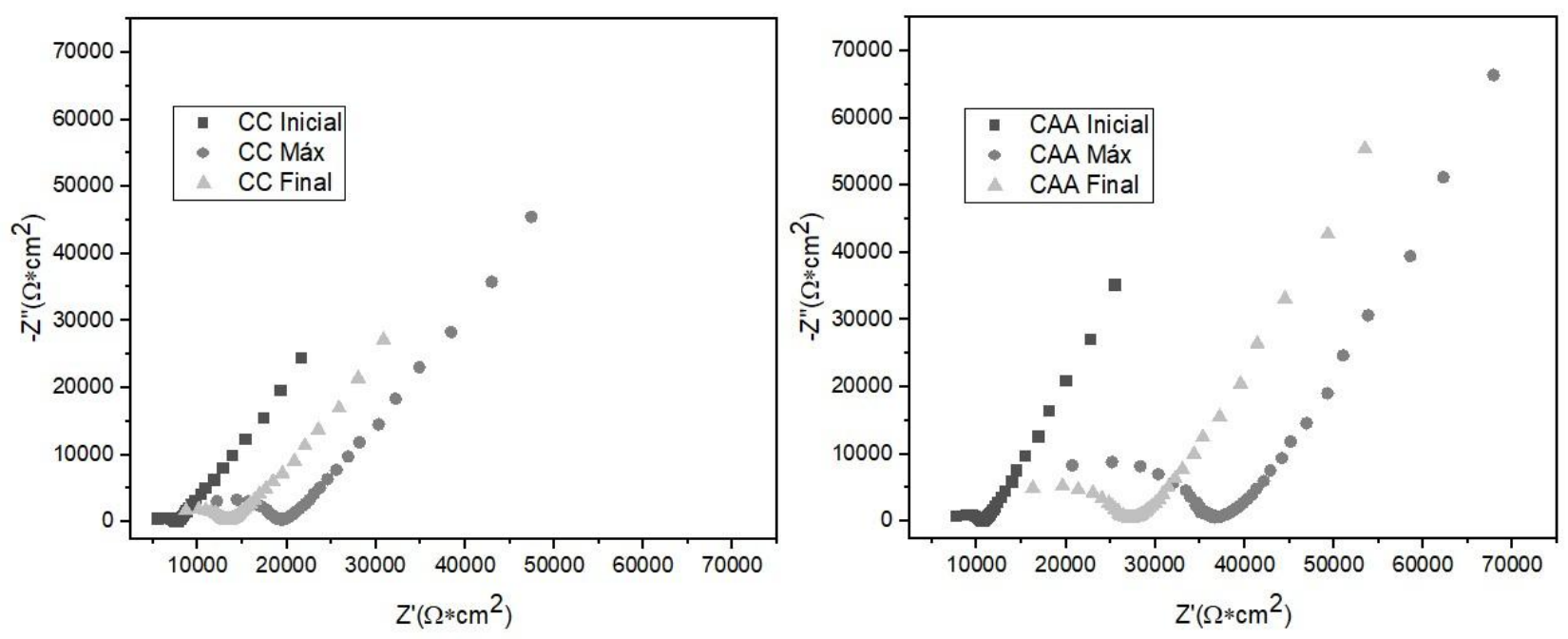

Figura 7: Diagrama de Nyquist para o concreto convencional (CC) (a) e para o concreto auto adensável (CAA) (b) para o primeiro ciclo, intermediario e final

Nos diagramas de Nyquist da Figura 7 podem ser visualizados semicírculos incompletos, correspondendo, provavelmente, às respostas da matriz do concreto e da interface pasta/aço ao potencial senoidal aplicado nas amostras. O resultado final representa aproximadamente dois anos do processo ( 80 ciclos).

Comparando os resultados da resistividade elétrica (impedância real (Z') verifica-se um comportamento resistivo nos dois tipos de concreto armado. Esta propriedade do material que define o quanto o material permite a passagem da corrente elétrica acaba corroborando com os resultados da porosidade. Os resultados do Z', impedancia real, demonstram um aumento superior a $90 \%$ da resistividade elétrica do CAA em relação ao CC.

Os resultados da degradação ficam evidentes pela redução da resitividade elétrica onde a resistividade de polarização máxima para o concreto convencional foi de $20100,5\left(\Omega^{*} \mathrm{~cm}^{2}\right)$ reduzindo para $13700\left(\Omega^{*} \mathrm{~cm}^{2}\right)$ enquanto para o concreto autoadensável foi de $38030 \Omega * \mathrm{~cm}^{2}$ para $27100\left(\Omega * \mathrm{~cm}^{2}\right)$. Os valores iniciais da resistividade de polarização são de $7511\left(\Omega * \mathrm{~cm}^{2}\right)$ para o concreto convencional e de $10546,2\left(\Omega * \mathrm{~cm}^{2}\right)$ para o concreto auto adensável considerando uma área de exposição de $35 \mathrm{~cm}^{2}$.

O aumento da resistividade elétrica analisados na Figura 7 corresponde a melhora das propriedades da matriz cimentícia mesmo submetida ao processo de envelhecimento para ambos os concretos podem ser verificadas através dos diagramas de Bode (Figura 8 e 9). 

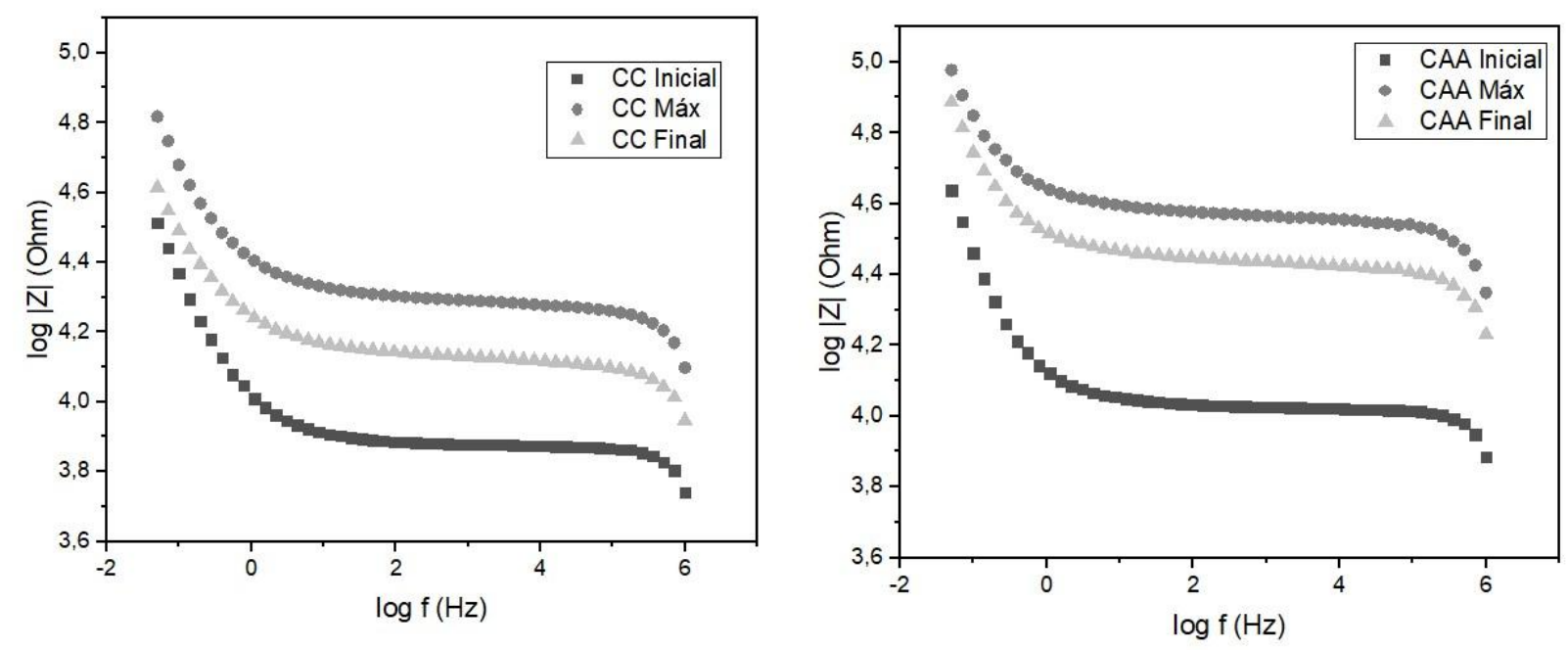

Figura 8: Diagrama de Bode para para a análise de EIS para o concreto convencional (CC) (a) e para o concreto auto adensável (CAA) (b) para os ciclos inicial, intermediário e final

Conforme o diagrama de Bode (Figura 8) para as regiões de alta frequência (acima de $10^{2} \mathrm{~Hz}$ ) verifica-se o aumento da resistência média devido ao processo de cura com a idade atingindo um valor máximo (CC Máx e CAA Máx) e depois reduzindo sendo esta redução bem menos expressiva no CAA.

Este aumento pode representar a melhora das propriedades da matriz cimentícia ao longo do tempo, decorrente das reações de formação dos produtos de hidratação do cimento, os quais preenchem os vazios e os poros, proporcionando o isolamento e a conseqüente proteção da armadura. Devido às condições de envelhecimento acelerado houve a redução na resistividade.

Nos diagramas de Bode (Figura 8), podem ser observadas três regiões: a da matriz do concreto e a da interface pasta/aço, que apareceram na extremidade à esquerda e à direita, respectivamente, e uma região entre as duas primeiras com um grande plateau resistivo, que correspondeu ao valor da resistência elétrica do concreto (Rc).
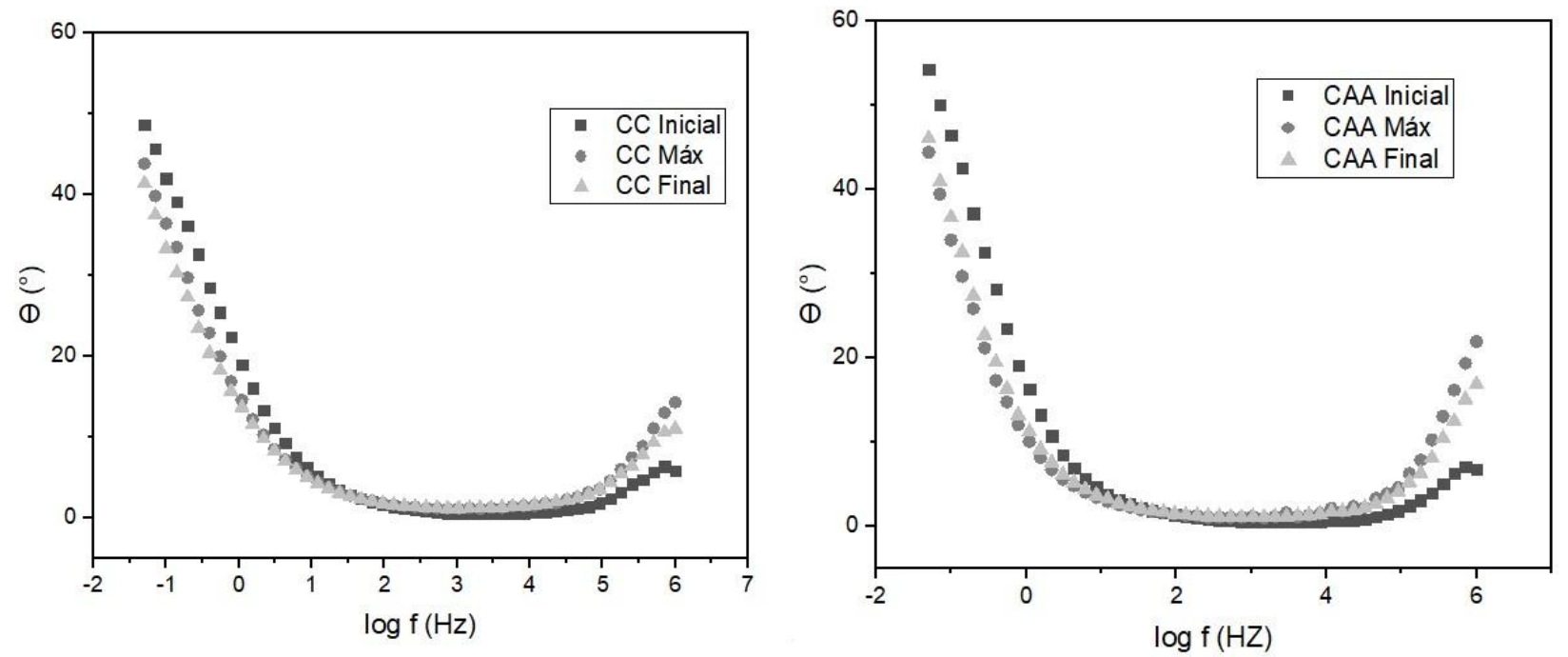

Figura 9: Diagrama de Bode para para a análise de EIS para o concreto convencional (CC) (a) e para o concreto auto adensável (CAA) (b) para os ciclos inicial, intermediário e final 
Na Figura 9 para a região de frequências intermediárias $\left(10^{1}\right.$ a $\left.10^{-1} \mathrm{~Hz}\right)$ no qual o ângulo de fase está representando uma curva ascendente foi possível verificar o comportamento capacitivo da dupla camada barra/matriz novamente evidenciando o aumento da resistência da interface entre a pasta de cimento e o filme passivo da armadura. Na região de baixas frequencias $\left(<10^{-1} \mathrm{~Hz}\right)$ verifica-se o aumento da resistência à tranferencia de carga para o concreto CAA se comparado com o convencional com uma tendência ao comportamento resistivo para CAA. O aumento da curva (Figura 9) em aproximadamente $45^{\circ}$, é uma indicação da difusão semi-infinita de espécies como íons de cloreto através de a camada de óxido e em direção ao eletrodo, juntamente com a carga reações de transferência

Baseado nos resultados da resistividade elétrica ao longo dos ciclos e períodos estudados, verifica-se que após em torno de 70 ciclos (CC máx e CAA máx) ocorre uma degradação da resistividade do material (CC Final e CAA Final). Esta redução na impedancia real é mais representativa no concreto convencional sendo confirmada pelos diagramas de Bode. Os resultados finais encontrados da impedância real foi uma redução menor a $25 \%$ no concreto autoadensável. Este resultado da degradação da resitência elétrica no concreto autoadensável é inferior se comparada ao concreto convencional.

\section{CONCLUSÕES}

O objetivo deste artigo foi analisar a degradação de dois tipos de concreto frente a penetração de cloretos em relação a sua resistividade elétrica, que mede indiretamente a durabilidade do material. Verifica-se que devido aos resultados encontrados no estudo o concreto auto adenável pode ser considerado com melhor durabilidade em meios salinos que o concreto convencional já que neste trabalho foi considerado amostras de concreto de mesma classe de resistência mecânica e mesma relação água/cimento.

A caracterização por EIS desenvolvida neste estudo para ambos os concretos nas mesmas condições mostram um aumento inicial da resitividade elétrica com o tempo de exposição mostrando um comportamento resistivo da pasta cimentícia. Como as amostras estão sendo submetidas ao processo de envelhecimento, após um período aproximadamente de 1 ano e meio, observa-se uma redução nos resultados da impedância real se comparado com os os ciclos anteriores, demostrando a degradação do material e surgindo a possibilidade e susceptibilidade a corrosão de armadura.

\section{AGRADECIMENTOS}

Agradecemos a Universidade Federal de Pelotas, ao Progama de Pos Graduação em Ciência e Engenharia de Materiais (PPGCEM), ao centro de microscopia eletrônica do sul (CEMESUL) ao Laboratório de Filmes Finos e Novos Materiais ( LAFFIMAT) e a Deakin University pela constribuições.

\section{REFERÊNCIAS}

ALEXANDER, M.; BEUSHAUSEN, H. Durability, service life prediction, and modelling for reinforced concrete structures-review and critique. Cement and Concrete Research, v. 122, p. 17-29, 2019.

ANGST, U. M., Elsener, B., Larsen, C. K., \& Vennesland, Ø.. Chloride induced reinforcement corrosion: Electrochemical monitoring of initiation stage and chloride threshold values. Corrosion Science, v. 53, n. 4, p. 1451$1464,2011$.

ASSIE, S.; ESCADEILlAS, G.; WALLER, V. Estimates of self-compacting concrete 'potential' durability. Construction and Building Materials, v. 21, n. 10, p. 1909-1917, 2007.

ASSOCIAÇÃO BRASILEIRA DE NORMAS TÉCNICAS. NBR 12655: Concreto de cimento Portland - Preparo, controle, recebimento e aceitação - Procedimento. Rio de Janeiro, 2015.

ASSOCIAÇÃO BRASILEIRA DE NORMAS TÉCNICAS. NBR 15823-1: Concreto autoadensável Parte 1: Classificação, controle e recebimento no estado fresco. Rio de Janeiro, 1998.

ASSOCIAÇÃO BRASILEIRA DE NORMAS TÉCNICAS. NBR 5738: Concreto - Procedimento para moldagem e cura de corpos de prova. Rio de Janeiro, 2016. 
ASSOCIAÇÃO BRASILEIRA DE NORMAS TÉCNICAS. NBR 5739: Concreto - Ensaio de compressão de corpos de prova cilíndricos. Rio de Janeiro, 2018.

ASSOCIAÇÃO BRASILEIRA DE NORMAS TÉCNICAS. NBR 6118: Projeto de Estruturas de concreto Procedimento. Rio de Janeiro, 2014.

ASSOCIAÇÃO BRASILEIRA DE NORMAS TÉCNICAS. NBR 67: Concreto - Determinação da consistência pelo abatimento do tronco de cone. Rio de Janeiro, 1998.

ASSOCIAÇÃO BRASILEIRA DE NORMAS TÉCNICAS. NBR 9779: Argamassa e concreto endurecidos Determinação da absorção de água por capilaridade. Rio de Janeiro, 2012.

BALONIS, M.; SANT, G.; ISGOR, O. B. Mitigating steel corrosion in reinforced concrete using functional coatings, corrosion inhibitors, and atomistic simulations. Cement and Concrete Composites, v. 101, p. 15-23, 2019.

BRAGANÇA, M. O., PORTELLA, K. F., BONATO, M. M., MARINO, C. E. Electrochemical impedance behavior of mortar subjected to a sulfate environment-A comparison with chloride exposure models. Construction and Building Materials, v. 68, p. 650-658, 2014.

BERTOLINI, L. Materiais de Construção: patologia, reabilitação, prevenção. São Paulo: Oficina de Textos. 2016.

BOURENANE, N.; SEDIRA, N.; HAMLAOUI, Y. Corrosion Behavior of Reinforcing Steel in Concrete Elements in Alkaline Medium. Journal of Building Materials and Structures, v. 6, n. 2, p. 50-63, 2019.

BUSARI, A. A.; AKINMUSURU, J. O.; DAHUNSI, B. I. Review of Sustainability in Self-compacting Concrete: the Use of Waste and Mineral Additives as Supplementary Cementitious Materials and Aggregates. Portugaliae. Electrochimica Acta, v. 36, n. 3, p. 147-162, 2018.

DE SCHUTTER, G; BARTOS, P. J. M.; DOMONE, P. GIBBS, J. Self-Compacting Concrete, Whittles Publishing, 2008, 288p.

FERREIRA, P. R. R. Análise da indução da corrosão por cloretos em concretos armados com adição de resíduo de tijolo moído a partir de ensaios acelerados. 2015. 229 f. Dissertação de Mestrado. Universidade Federal de Pernambuco. Recife, PE, Brasil, 2015.

GARDONI P; MURPHY C. Society-based design: promoting societal well-being by designing sustainable and resilient infrastructure. Sustainable and Resilient Infrastructure p:1-16, May 2018.

GUIRGUIS, B., SHEHATA, M. H., DUCHESNE, J., FOURNIER, B., DURAND, B., RIVARD, P. The application of a new oxidation mortar bar test to mixtures containing different cementing systems. Construction and Building Materials, v. 173, p. 775-785, 2018.

HELENE, P.; GUIGNONE, G.; VIEIRA, G.; RONCETTI, L.; MORONI, F. Avaliação da penetração de cloretos e da vida útil de concretos autocicatrizantes ativados por aditivo cristalino. RIEM-IBRACON Structures and Materials Journal, v. 11, n. 3, 2018.

HELENE, P.; TERZIAN, P. Manual de dosagem e Controle do concreto. São Paulo: Pini, 1992.

JIANG, C.; WU, Y.; DAI, M. Degradation of steel-to-concrete bond due to corrosion. Construction and Building Materials, v. 158, p. 1073-1080, 2018.

LI, D.; WEI, R.; LI, L.; GUAN, X.; MI, X. Pitting corrosion of reinforcing steel bars in chloride contaminated concrete. Construction and Building Materials, v. 199, p. 359-368, 2019.

LI, K.; ZHANG, D.; Li, Q.; FAN, Z. Durability for concrete structures in marine environments of HZM project: Design, assessment and beyond. Cement and Concrete Research, v. 115, p. 545-558, 2019. 
LUPING, T.; GULIKERS, J. On the mathematics of time-dependent apparent chloride diffusion coefficient in concrete. Cement and concrete research, v. 37, n. 4, p. 589-595, 2007.

MEDEIROS-JUNIOR, R. A.; GANS, P. S. Efeito da adição de pozolana na resistividade elétrica superficial do concreto. Revista de Engenharia e Pesquisa Aplicada, v. 2, n. 3, 2017.

OKAMURA, H. Self-Compacting High Performance. Concrete International, Vol. 19, No. 7, pp. 50-54, ACI, July. 1997.

REAL, L. V.; OLIVEIRA, D. R. B.; SOARES, T.; MEDEIROS, M. H. F.. Método colorimétrico por aspersión de nitrato de plata para laevaluación de lapenetración de clorurosen concreto: estado del arte. Revista Alconpat, v. 5, n. 2, p. 151-161, 2015.

RIBEIRO, R. R. J.; DIÓGENES, H. J. F.; NOBREGA, M. V.; DEBS, A. E. A survey of the mechanical properties of concrete for structural purposes prepared on construction sites. Revista IBRACON de Estruturas e Materiais, v. 9, n. 5, p. 722-744, 2016.

ROVNANÍK, P., KUSÁK, I., BAYER, P., SCHMID, P., FIALA, L. Comparison of electrical and self-sensing properties of Portland cement and alkali-activated slag mortars. Cement and Concrete Research, v. 118, p. 84-91, 2019.

SERRALHEIRO, M. I.; DE BRITO, J.; SILVA, A. Methodology for service life prediction of architectural concrete facades. Construction and Building Materials, v. 133, p. 261-274, 2017

SHI, W., CHEN, Y., LIU, P., XU, D. Corrosion Investigation of Reinforced Concrete Based on Piezoelectric Smart Materials. Materials, v. 12, n. 3, p. 519, 2019.

SOHAIL, M. G., KAHRAMAN, R., ALNUAIMI, N. A., GENCTURK, B., ALNAHHAL, W., DAWOOD, M., BELARBI, A. Electrochemical behavior of mild and corrosion resistant concrete reinforcing steels. Construction and Building Materials, v. 232, p. 117205, 2020.

STANDARD, A. S. T. M. G1-03. Standard practice for preparing, cleaning and evaluating corrosion test specimens", American Society for Testing and Materials, 2003.

STEFANONI, M.; ANGST, U.; ELSENER, B. Corrosion rate of carbon steel in carbonated concrete-A critical review. Cement and Concrete Research, v. 103, p. 35-48, 2018.

TIAN, Y.; WANG, P.; ZHAO, T.; MA, Z.; JIN, Z.; ZHAO, H. Influence of Water-Repellent Treatment with Silicon Resin on Properties of Concrete. Advances in Materials Science and Engineering, v. 2019, 2019.

TUTIKIAN, B. F.; DAL MOLIN, D. C. Concreto auto-adensável. São Paulo: PINI, 2008.

VAN ZIJL GPAG, P. SC. A Novel Link of The Time Scale in Accelerated Chloride-Induced Corrosion Test in Reinforced SHCC. Construction and Building Materials, v. 167, p. 15-19, 2018.

VEDALAKSHMI, R., SARASWATHY, V., SONG, H. W., PALANISWAMY, N. Determination of diffusion coefficient of chloride in concrete using Warburg diffusion coefficient. Corrosion Science, v. 51, n. 6, p. 1299-1307, 2009.

WEI, Y.; GUO, W.; LIANG, S. Chloride Ingress in Internally Cured Concrete under Complex Solution. Journal of Materials in Civil Engineering, v. 30, n. 4, p. 04018037, 2018.

WU, L.; LI, W.; YU, X. Time-dependent chloride penetration in concrete in marine environments. Construction and Building Materials, v. 152, p. 406-413, 2017.

ZHANG, Y.; ZHANG, M.; YE, G. Influence of moisture condition on chloride diffusion in partially saturated ordinary Portland cement mortar. Materials and Structures, 51, n. 2, p. 36, 2018. 